\title{
Dosimetric Study for the Feasibility of Carotid Sparing Intensity Modulated Radiation Therapy for Early Glottic Cancer
}

\author{
Preeya Vasanthakumary ${ }^{1}$, Anilkumar Karumathil'2, Gargy Anjolian David ${ }^{3}$, \\ Sivaramakrishnan Ramachandran ${ }^{4}$, Anjana Sasikumar Nair ${ }^{5}$ \\ 1, 2, 3,4 Department of Radiation Oncology, Government Medical College, Kottayam, Kerala, India. \\ ${ }^{5}$ Amrita Institute of Medical Sciences (AIMS), Kochi, Kerala, India.
}

\section{ABSTRACT}

\section{BACKGROUND}

The primary objective of this study is a dosimetric comparison for the feasibility of carotid sparing intensity modulated radiation therapy (CS-IMRT) for early glottis cancer. The planning target volume coverage and the spinal cord dose are compared in the 3-dimensional conformal radiotherapy (3DCRT) and IMRT plans.

\section{METHODS}

In this single reconcile study twenty-five patients who had been already treated for early-stage glottis cancer (T1, T2N0M0) from June 2015 to June 2021 with 52.5 Gy/15 fractions were included. All patients were simulated in supine position with an orfit shell and treatment planning computed tomography scans were obtained from the vertex to sternal angle with a 3-mm slice thickness. Treatment was executed with 3-DCRT plan and comparison was made with the newly created IMRT plan. The analysis was made regarding mean dose to the carotid arteries, volume of carotid artery receiving more than 25 Gy (V25), 35 Gy (V35), 50 Gy (V50), planning target coverage and doses to organ at risk.

\section{RESULTS}

The dose to carotids was significantly higher in the 3-DCRT plan compared with the IMRT plan ( $\mathrm{p}=0.004)$. For target coverage the volume receiving $95 \%$ of the dose was significantly higher in IMRT plan $(\mathrm{p}=.001)$. The dose to the spinal cord was higher-in the IMRT plan $(\mathrm{p}=.001)$ compared to 3 -DCRT plan.

\section{CONCLUSIONS}

IMRT maintains target volume coverage with significant reduction in carotid artery dose. This reduces the incidence of cerebrovascular accidents by decreasing the radiation induced carotid artery stenosis, thereby improving the quality of life of patients.

\section{KEY WORDS}

CS-IMRT, 3D-CRT, Mean Carotid Dose
Corresponding Author: Dr. Preeya Vasanthakumary, Advaitham, Vattackatt, Kaviyoor, Tiruvalla,

Kerala, India.

E-mail: preeya.anilkumar7@gmail.com

DOI: $10.14260 /$ jemds/2022/19

How to Cite This Article: Vasanthakumary P, Karumathil A, David GA, et al. Dosimetric study for the feasibility of carotid sparing intensity modulated radiation therapy for early glottic cancer. J Evolution Med Dent Sci 2022;11(01):98-102, DOI: $10.14260 /$ jemds $/ 2022 / 19$

Submission 16-12-2021,

Peer Review 11-01-2022,

Acceptance 17-01-2022,

Published 25-01-2022.

Copyright (c) 2022 Preeya Vasanthakumary et al. This is an open access article distributed under Creative Commons Attribution License [Attribution 4.0 International (CC BY 4.0)] 


\section{BACKGROUND}

Approximately $30-40 \%$ of all laryngeal cancer are constituted by early glottic cancers. ${ }^{1}$ Early laryngeal cancer has shown excellent local control and survival rates with radical radiation or surgical treatment. Lymph node metastasis is rare in case of early glottis cancer, so localized radiotherapy to larynx can successfully treat early glottis cancer with voice quality preservation and low toxicity levels. So, radiation is the preferred treatment option among these patients. ${ }^{2}$ Patients treated with radiation for head and neck cancers require therapeutic or elective nodal irradiation for nodal metastases. But risk of nodal metastases for early glottic cancer is negligible. This enables to tailor radiation target avoiding nearby carotid arteries. This dosimetric distinction is allowed by intensity modulated radiation therapy (IMRT). The opposed lateral beams by conventional radiation technique give full or greater radiation dose to carotids resulting in increased cerebrovascular accidents like ischemic strokes or transient ischemic attacks. Gujral et al. ${ }^{3}$ reported that radiation increase the thickness of carotid intima-media leading to carotid artery stenosis and thereby cerebrovascular accidents. The primary risk factor for laryngeal cancer is tobacco use which together with radiation increase the risk of cerebrovascular morbidity and mortality. In the context, techniques minimizing cerebrovascular risk are appealing. Hence, this retrospective study is aimed for the dosimetric comparison for the feasibility of carotid sparing IMRT in early glottis cancer.

The primary objective of our study was to make a dosimetric comparison of dose received by carotids in the 3D-CRT and IMRT plans. The secondary objective was to compare PTV coverage and the dose received by the spinal cord.

\section{METHODS}

This single reconcile study design was conducted in the Department of Radiation Oncology of a tertiary cancer centre located in the Central part of the state. Twenty-five patients with early glottis cancer (T1, T2 N0M0) treated with the radiation dose schedule of $52.5 \mathrm{~Gy}$ in 15 fractions by 3D CRT plan between June 2015 and June 2021 were included in the study. These patients were staged with direct laryngoscopy, computed tomography (CT scans), tissue biopsy and chest Xray. The study was initiated after approval of institutional review board with IRB No. 72/2021.

\section{Sample Size}

Based on the results of mean and SD of planning target volume $95 \%$ of patients treated with 3-DCRT $\left(95.5+\_1\right)$ and IMRT $(96+1$ ) for early glottis cancer, observed in earlier publication of Mohamed ASR et al. ${ }^{4}$ and with $80 \%$ power and $95 \%$ confidence limit, sample size is 34 .

$\mathrm{N}=\left(\mathrm{S}_{1}{ }^{2}+\mathrm{S}_{2}{ }^{2}\right) *\left(\mathrm{Z}_{1-\alpha / 2}+\mathrm{Z}_{1-\beta / 2}\right)^{2} /(\mathrm{X} 1-\mathrm{X} 2)^{2}$

$=\left(1^{2}+0.3^{2}\right)^{*}(1.96+0.84)^{2} /(96-95.5)^{2}$

$=1.09 *[7.84 / 0.25]$

$=34.18$

$=34$

\section{Inclusion Criteria}

All patients who have undergone radiotherapy for early glottic cancer by hypofractionated 3 DCRT plan in linear accelerator in the Department of Radiation Oncology.

\section{Exclusion Criteria}

1. Patients who have undergone radical radiotherapy in Co 60 teletherapy machine for early glottic cancer.

2. Patients who have not completed the full course of radical radiotherapy.

Planning CT images of $3 \mathrm{~mm}$ slices were available in the Eclipse treatment planning system. All these patients had already received treatment by hypofractionated schedule of 52.5 Gy in 15 fractions over 3 weeks with 3-D CRT plan between June 2015 and June 2021. A new IMRT plan was created for the same patients after contouring the carotids.

Two plans (3-DCRT and IMRT) which were created for each patient on Eclipse treatment planning system with triple Aalgorithm [Anisotropic Analytical Algorithm (AAA)] were compared. The data derived from these plans were recorded for the comparative study. The comparison between conventional and IMRT plans were done using generalized equivalent uniform dose and dose-volume parameters for infield carotid arteries, target volumes, and organs at risk.

All patients received treatment by 3D-CRT with $6 \mathrm{MV}$ photons which was delivered by Clinac linear accelerator, Varian Medical Systems, Palo Alto CA having 40 paired multileaf collimators of leaf width $1 \mathrm{~cm}$ at the isocentre.

Clinical target volume (CTV) extended from thyroid notch superiorly to the lower border of cricoid cartilage inferiorly, posteriorly to include arytenoid cartilage, posterior commissure and anteriorly and laterally to include the anterior commissure. Thyroid cartilage was excluded. PTV was generated by adding $0.5 \mathrm{~cm}$ to CTV. The planning target coverage was normalized such that $100 \%$ of the PTV received more than $95 \%$ of the prescription dose.

The carotid arteries and spinal cord were delineated as organ at risk (OAR). The carotid OAR is defined as the extracranial extent of carotid artery from $2.5 \mathrm{~cm}$ superior to the hyoid bone to aortic arch on left /brachiocephalic trunk on the right, inferiorly. The carotid artery diameter (approximately $6.5 \mathrm{~mm}$ ) changes $15 \%$ during cardiac cycle. A $15 \%$ increase of $6.5 \mathrm{~mm}$ is $0.98 \mathrm{~mm}$, so a further $1 \mathrm{~mm}$ was added as planning at risk volume (PRV). The mean dose received by the carotids (MCD), volume receiving 25 Gy, 35Gy \& 50 Gy (V25, V35, V50) was recorded from the two treatment plans and they were compared.

The spinal cord dose received by the two treatment plans were noted for comparison.

The spinal cord was contoured from foramen magnum to $2.5 \mathrm{~cm}$ below PTV. Spinal cord PRV of $3 \mathrm{~mm}$ was added in all directions of the spinal cord organ at risk (OAR).

\section{Statistical Analysis}

All statistical data collected was entered into excel sheet and analysed by SPSS Software version 23. Continuous variables were expressed as mean \pm standard deviation. All quantitative variables were checked for normal distribution within each category of explanatory variable by using visual 
inspection of Q-Q plots and histograms. Shapiro Wilk test was also conducted to assess normal distribution. Shapiro Wilk test $p$ value of $>0.05$ was considered as normal distribution. Since this a retrospective study all patients (twenty-five) with early glottis cancer treated with hypofractionated radiation schedule of 52.5 Gy in 15 fractions from June 2015 to June 2021 were included in the study. Mann Whitney U Test, the non-parametric test of independent $t$ test was used to compare two independent samples. Wilcoxon rank sum test was used for continuous variable comparison between cohorts. $\mathrm{P}$ values $<0.05$ at $95 \%$ confidence intervals was considered as statistically significant.

\section{RESULTS}

This comparative dosimetric study was done by creating a new IMRT plan for the same patients who had already completed treatment and now on follow up.

The median age was 65 years with $96 \%$ males and $4 \%$ females. 22(88\%) were smokers, $20(80 \%)$ were alcoholic. Majority of the patients were of T1a (68\%) substage. 32\% were $\mathrm{T} 1 \mathrm{~b}$ and $\mathrm{T} 2$ substages, each $16 \%$ respectively. The risk factors for cerebrovascular events noted were hypercholesterolemia in $4(16 \%)$, hypertension in $5(20 \%)$ and diabetes in $2(8 \%)$.

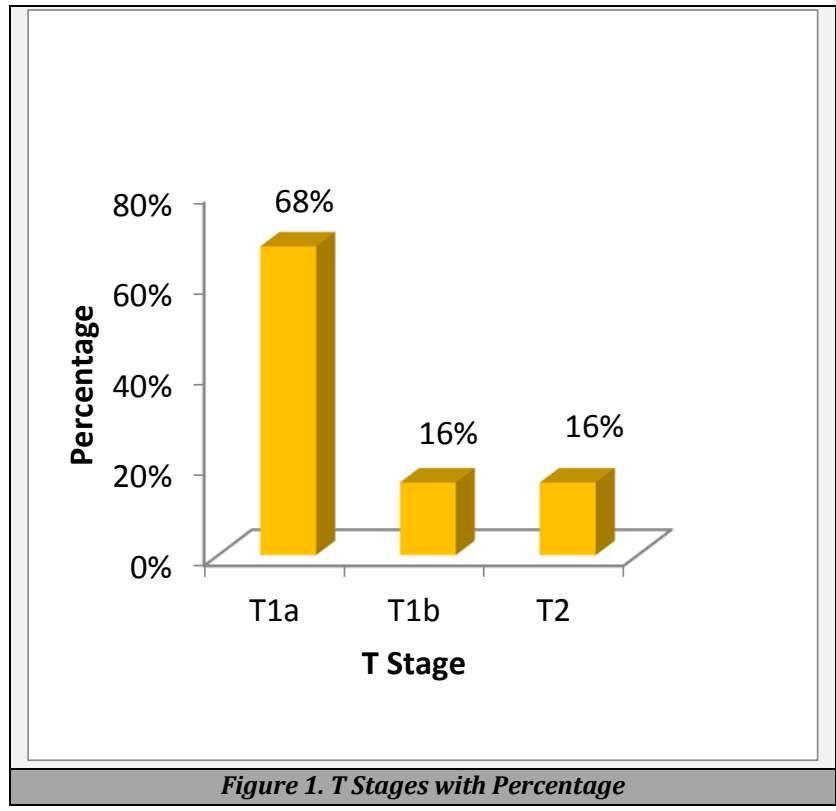

The median $95 \%$ PTV dose was 49.85 Gy $(94.95 \%$ of the prescribed dose) for 3-DCRT and for IMRT 52.2 Gy (99.4\% of the prescribed dose). The mean of the average doses of carotid artery segments in the region of the PTVfor 3-DCRT and IMRT were 46.7 Gy (EQD 2 -51.3 Gy)and 43.1Gy (EQD 246.2 Gy),respectively. The magnitude of carotid sparing among the cohorts varied with a minimum carotid dose of 35.3 Gy (EQD $2-36.3 \mathrm{~Gy})$ for 3-DCRT and 31.3 Gy (EQD $2-31.5)$ for IMRT. Volume of carotid artery segment receiving 25Gy, 35 Gy and 50 Gy (V25, V35 and V50) by 3DCRT were 5.09cc, $4.9 \mathrm{cc}$ and $3.8 \mathrm{cc}$ respectively. V25,V35 and V50 for carotid by IMRT were $4.8 \mathrm{cc}, 4.1 \mathrm{cc}$, and $1.9 \mathrm{cc}$ respectively.V50 in the 3DCRT group was statistically significantly higher than the IMRT group ( $\mathrm{U}=103.5, \mathrm{p}=.001)$.
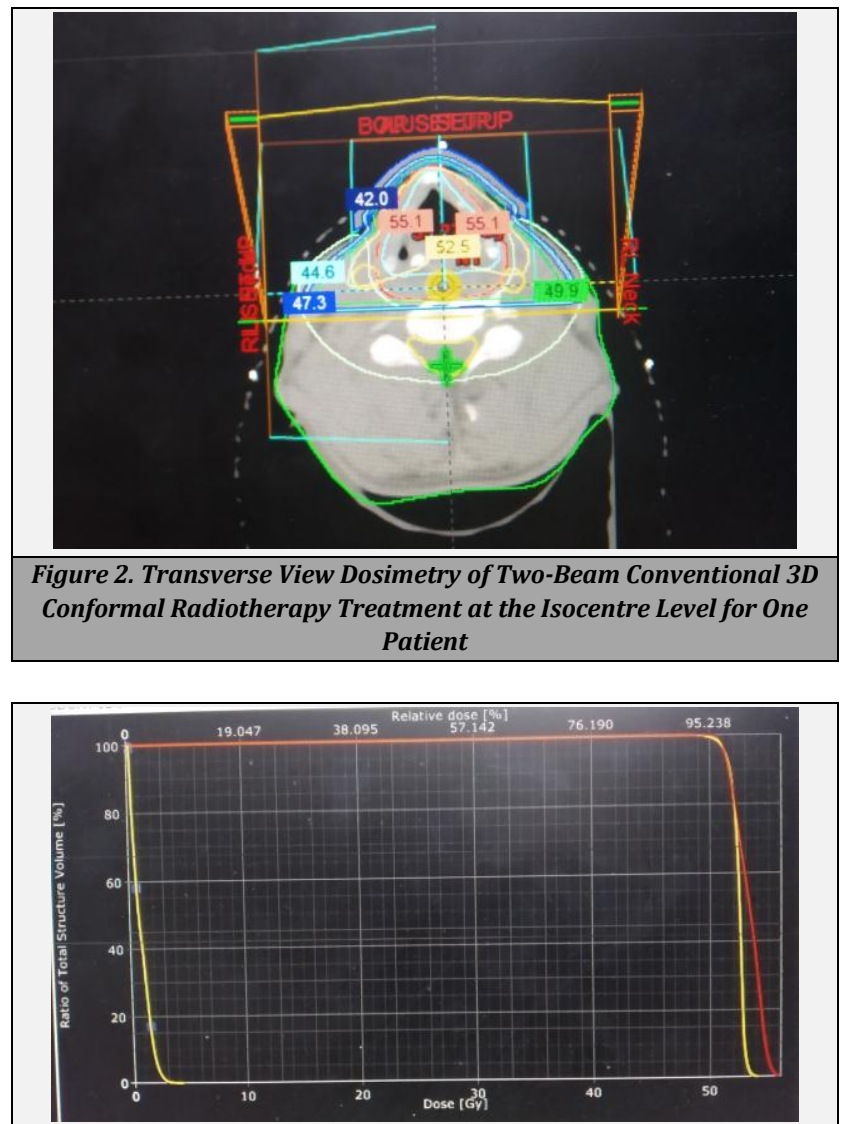

Figure 3. Dose-Volume Histogram for 3D Planning Techniques. Contours for PTV (red lines), Planning Targets at Risk Volume of Carotid Arteries (yellow line -right) and Spinal Cord (yellow line-left)

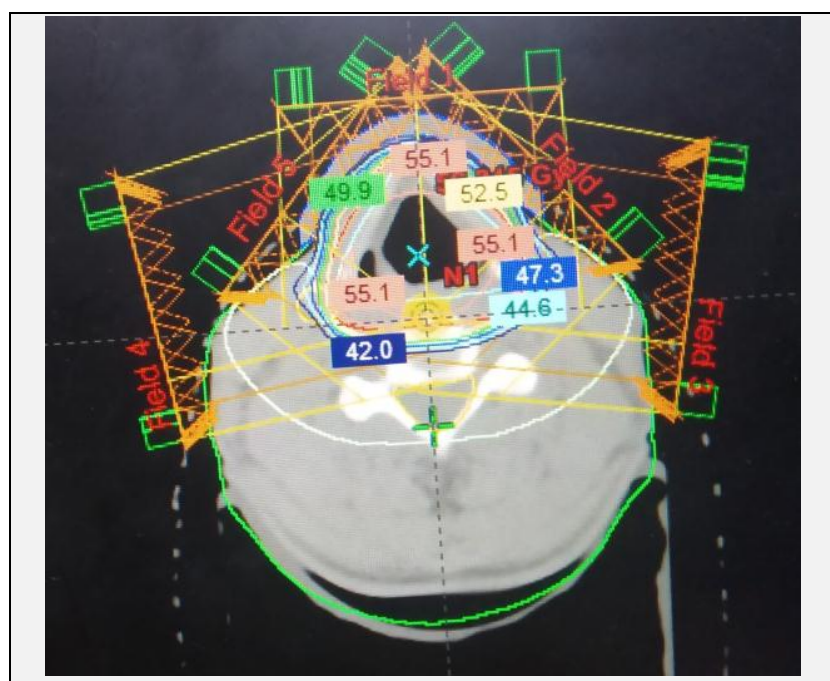

Figure 4. Transverse View Dosimetry Comparison Intensity-Modulated Radiotherapy (IMRT) Treatment at the Isocentre Level for One Patient

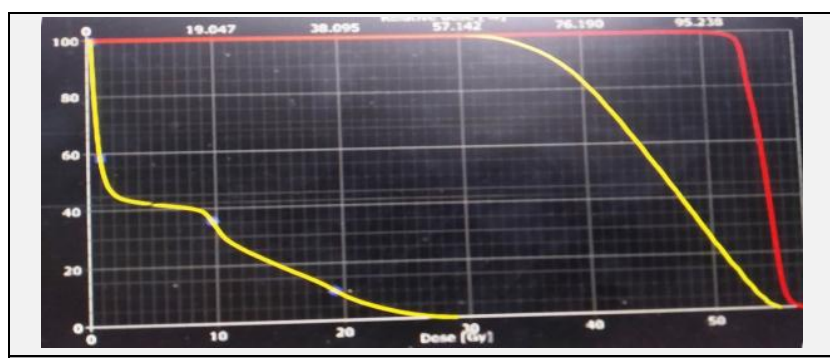

Figure 5. Dose-Volume Histogram for IMRT Planning Techniques. Contours for PTV (red lines), Planning Targets at Risk Volume of Carotid Arteries (yellow line -right) and Spinal Cord (yellow line-left) 


\begin{tabular}{|cccccc|}
\hline \multicolumn{5}{c}{ Descriptive Statistics } \\
& N & Minimum & Maximum & Mean & Std. Deviation \\
AGE & 24 & 42 & 81 & 62.58 & 10.778 \\
MCD 3D & 25 & 35.3000 & 53.7000 & 46.896800 & 5.2628341 \\
MCD IM & 25 & 31.7000 & 51.3000 & 43.220000 & 9.7247108 \\
V25 3D & 25 & .1000 & 14.3000 & 5.430400 & 2.6790538 \\
V25 IM & 25 & .1000 & 14.0000 & 5.241200 & 2.5838075 \\
V353D & 25 & .1000 & 14.0000 & 5.267200 & 2.6090236 \\
V35 IM & 25 & .0560 & 13.5000 & 4.517440 & 2.4625861 \\
V50 3D & 25 & .0500 & 12.3000 & 4.403200 & 2.2976541 \\
V50 IM & 25 & .0000 & 12.0000 & 2.293920 & 2.4533323 \\
MU 3D & 25 & 379 & 436 & 403.60 & 12.196 \\
MU IM & 25 & 493 & 875 & 662.56 & 83.939 \\
SC D0SE 3D & 25 & .8370 & 8.4000 & 2.811480 & 1.8136356 \\
SCD0SEIM & 25 & 5.2400 & 34.5000 & 20.889600 & 5.9956682 \\
D0SE953D & 24 & 5.09 & 51.90 & 46.2496 & 9.87668 \\
D0SE95IM & 24 & 51.2 & 53.2 & 52.304 & .5146 \\
\hline Table 1. Descriptive Statistics of Continuous Variables are Given below
\end{tabular}

The mean monitor units required to deliver the radiation dose of 52.5 Gy in 15 fractions by 3-DCRT is 403MU and that by IMRT was 662 MU. Thus, the time utilized was more for IMRT than for 3-DCRT for same radiation dose. ( $U=0.000$, p $=.001$ ). The mean spinal cord dose was $2.8 \mathrm{~Gy}$ for 3 -DCRT and 20.9 Gy for IMRT. $(U=4, p=.001)$.

\begin{tabular}{|c|c|c|c|c|}
\hline & Group & $\mathbf{N}$ & Mean Rank & Sum of Ranks \\
\hline \multirow{3}{*}{ MCD } & 3DCRT & 25 & 31.46 & 786.50 \\
\hline & IMRT & 25 & 19.54 & 488.50 \\
\hline & Total & 50 & & \\
\hline \multirow{3}{*}{ V25 } & 3DCRT & 25 & 26.22 & 655.50 \\
\hline & IMRT & 25 & 24.78 & 619.50 \\
\hline & Total & 50 & & \\
\hline \multirow{3}{*}{ V35 } & 3DCRT & 25 & 28.54 & 713.50 \\
\hline & IMRT & 25 & 22.46 & 561.50 \\
\hline & Total & 50 & & \\
\hline \multirow{3}{*}{ V50 } & 3DCRT & 25 & 33.86 & 846.50 \\
\hline & IMRT & 25 & 17.14 & 428.50 \\
\hline & Total & 50 & & \\
\hline \multirow{3}{*}{ MU } & 3DCRT & 25 & 13.00 & 325.00 \\
\hline & IMRT & 25 & 38.00 & 950.00 \\
\hline & Total & 50 & & \\
\hline \multirow{3}{*}{ SCDOSE } & 3DCRT & 25 & 13.16 & 329.00 \\
\hline & IMRT & 25 & 37.84 & 946.00 \\
\hline & Total & 50 & & \\
\hline \multirow{3}{*}{ D0SE95 } & 3DCRT & 25 & 13.36 & 334.00 \\
\hline & IMRT & 25 & 37.64 & 941.00 \\
\hline & Total & 50 & & \\
\hline \multicolumn{5}{|c|}{$\begin{array}{c}\text { Table 2. Dosimetric Data of Each Variables -Target Volume and } \\
\text { Organs at Risk in Intensity-Modulated Radiotherapy (IMRT) and 3D } \\
\text { Conformal Radiotherapy Plans Shown as Rank Table }\end{array}$} \\
\hline
\end{tabular}

\begin{tabular}{|c|c|c|c|c|c|c|c|}
\hline & MCD & V25 & V35 & V50 & MU & SCDOSE & DOSE95 \\
\hline $\begin{array}{c}\text { Mann- } \\
\text { Whitney } \\
\text { U }\end{array}$ & 163.500 & 294.500 & 236.500 & 103.500 & .000 & 4.000 & 9.000 \\
\hline $\begin{array}{c}\text { Wilcoxon } \\
\text { W }\end{array}$ & 488.500 & 619.500 & 561.500 & 428.500 & 325.000 & 329.000 & 334.000 \\
\hline $\mathrm{Z}$ & -2.892 & -.350 & -1.475 & -4.057 & -6.065 & -5.987 & -5.894 \\
\hline \begin{tabular}{|l} 
Asymp. \\
Sig. (2- \\
tailed) \\
\end{tabular} & .004 & .727 & .140 & .000 & .000 & .000 & .000 \\
\hline \multicolumn{8}{|c|}{ Table 3. Test Statistics } \\
\hline $\begin{array}{l}\text { Mann Wl } \\
\text { Wilcoxor } \\
\text { values < }\end{array}$ & $\begin{array}{l}\text { ey U Te } \\
\text { k sum } \\
\text { at } 95 \%\end{array}$ & $\begin{array}{l}\text { ndepen } \\
\text {-for con } \\
\text { ffidence }\end{array}$ & $\begin{array}{l}\text { dent } T \text { test } \\
\text { tinuous } v \\
\text { intervals }\end{array}$ & $\begin{array}{l}\text {-to compa } \\
\text { riable- con }\end{array}$ & $\begin{array}{l}\text { re two } \\
\text { mparis } \\
\text { ly signi }\end{array}$ & $\begin{array}{l}\text { epende } \\
\text { etweer } \\
\text { nt. }\end{array}$ & $\begin{array}{l}\text { mples. } \\
\text { orts. P }\end{array}$ \\
\hline
\end{tabular}

\section{DISCUSSION}

Carotid arteries lying in the lateral opposed parallel pair beam pathway for early glottis cancer receives relatively high doses almost identical to that of the target dose. FernándezAlvarez et al. ${ }^{5}$ reviewed the current aspects related to cerebrovascular events in irradiated patients of head and neck cancer. Carotid artery stenosis reported by them in such patients were 18 to $38 \%$ compared 0 to $9.2 \%$ among nonirradiated. The intima-media thickness of carotid wall induced by radiation is increased and it is detected by colour Doppler ultrasonography. Radiation-induced atherosclerosis is of accelerated and aggressive nature with a different biologic behaviour. Simonetti et $a .^{6}$ stated that the irradiated group had a higher plaque score and that bilateral plaque score was significantly influenced by age, hyperlipidaemia and radiation. The most affected site was internal carotid artery's fork as demonstrated by Eco Doppler examination. In their analysis age was inversely correlated with plaque score in irradiated patients more than 50 years old and positively correlated with plaque score in irradiated less than or equal to 41 years old. The 10-year incidence of cerebrovascular events was increased by $9 \%$ as reported by Smith et al. ${ }^{7}$ and 15 -year cumulative risk of stroke was $12 \%$ as stated by Dorresteijn et al. ${ }^{8}$ Thus the incidence of stroke was $5 \cdot 6$ times higher in irradiated compared to non-irradiated. Hypertension, smoking, hypercholesterolemia and diabetes mellitus were registered as other risk factors of stroke.

Carotid -sparing techniques with modified oblique field, IMRT, arc therapy or tomotherapy were employed with a view to reduce dose to the carotid artery while irradiating early glottic malignancy. Simplified IMRT planning algorithm with three fields and limited segments was the first reported CS-IMRT technique by Rosenthal et al. ${ }^{9}$ for 11 patients with T1-2 glottic cancer. Patients with long life expectation and with pre-existing vascular risk factors (smoking history, diabetes, and hypercholesterolemia) may be the most beneficial population. ${ }^{10}$

Zumsteg ZS et al.11 has demonstrated that local failure rates were similar with CS-IMRT and CRT with excellent carotid sparing. Riegel AC et al. ${ }^{12}$ did a comparative dosimetric study between volumetric modulated arc therapy and limited-angle static intensity-modulated radiation therapy for early-stage larynx cancer. The most effective carotid sparing with highest mean normal tissue dose was with full -arc planning. The next-best carotid sparing with lower normal tissue dose is with static IMRT. Whereas, anterior half-arc produced highest carotid artery dose comparable with conventional planning. Maximum dose of $<35$ Gy would be the reasonable dose to set a stringent constraint to produce a positive impact of CS-IMRT on future neurological events. ${ }^{3}$

The criticisms for IMRT for early glottis cancer (EGC) was made by Chakraborty et al. ${ }^{13}$ of Malabar Cancer centre, Kerala quoting the study of Chatterjee et al. and indicated that

1. Absolute dose delivered to the carotid is decided by final dose prescribed rather than its relative sparing.

2. They observed that Smith et al. reported $4.9 \%$ and Scott et al. $2.9 \%$ (crude risk) of ischemic stroke in irradiated patients. But Hong et al. in a recent analysis of surveillance epidemiology and end results data had indicated no difference of cerebrovascular accidents in EGC treated either with radiotherapy or surgery and hence, absolute magnitude is undoubtedly minimal. ${ }^{14}$

3. They also reported mean dose of even 15-16 Gy was not safe as quoted by Dorth et al. that hazard ratio for carotid artery stenosis was 1.4 for every $10 \mathrm{~Gy}$ increase in mean dose to carotid bulb.

4. Impact of intra fractional laryngeal motion on target dose delivery and treatment time were not commented which have a significant effect on instantaneous target dosage in IGRT. 
5. Trimming of PTV near carotid arteries was not warranted as planning target volume is the margin meant for inaccuracies in set up error and organ motion which shall not change near carotids.

Stating above criticism they have argued for cost effective conventional treatment in place of expensive helical tomotherapy for uncertain clinical gains.

The present study also demonstrated results as that of previous series quoted, ${ }^{4}$ with higher PTV coverage for V 95\% and lower carotid dose with IMRT. The increased integral dose and the more probable chances of geometrical misses by increased conformality were more for IMRT.

The spinal cord dose in our study was significantly higher with IMRT compared with 3D-CRT, but was well below the dose constraint specified for spinal cord.

\section{CONCLUSIONS}

Carotid sparing IMRT significantly lowers the radiation dose to the carotids with adequate PTV coverage compared to 3DCRT. This reduces the incidence of carotid artery stenosis and cerebrovascular events producing a positive influence in the quality of life.

\section{Limitations \\ Our study is limited because of its retrospective dosimetric comparison alone. A prospective comparative study with simultaneous comparison of two treatment plans carried out in patients with extended long-term data will derive a valid conclusion. The more conformal techniques like IMRT might increase the risk of geographic miss and subsequent local failure because of organ motion and/or uncertainties in target delineation and hence require further validation. Besides, outcomes for carotid artery effects and cancer control in both treatment cohorts require additional experience, larger cohorts, and longer follow-up. The carotid OAR dose constraint is lacking for most of the above- mentioned studies which needs to be clearly defined in future prospective studies. The formal voice quality assessment after treatment cannot be derived by this mere dosimetric comparative study.}

Data sharing statement provided by the authors is available with the full text of this article at jemds.com.

Financial or other competing interests: None.

Disclosure forms provided by the authors are available with the full text of this article at jemds.com.

\section{REFERENCES}

[1] Mendenhall WM, Mancuso AA, Amdur RJ, et al. Laryngeal cancer. In: Halperin EC, Brady LW, Perez CA, et al, eds. Perez and Brady's principles and practice of radiation oncology. $6^{\text {th }}$ edn. Philadelphia, PA: Lippincott Williams \& Wilkins 2013:850-68.

[2] Teshima T, Chatani M, Hata K, et al. Radiation therapy of early glottic cancer (T1N0M0); retrospective review of historical control. Rinsho Hoshasen 1989;34(13):1603-6.

[3] Gujral DM, Shah BN, Chahal NS, et al. Clinical features of radiation-induced carotid atherosclerosis. Clin Oncol (R Coll Radiol) 2014;26(2):94-102.

[4] Mohamed ASR, Smith BD, Smith JB, et al. Outcomes of carotid-sparing IMRT for T1 glottic cancer: comparison with conventional radiation. Laryngoscope 2020;130(1):146-53.

[5] Fernández-Alvarez V, López F, Suarez C, et al. Radiationinduced carotid artery lesions. Strahlenther Onkol 2018;194(8):699-710.

[6] Simonetti G, Pampana E, Di Poce I, et al. The role of radiotherapy in the carotid stenosis. Ann Ital Chir 2014;85(6):533-6.

[7] Smith GL, Smith BD, Buchholz TA, et al. Cerebrovascular disease risk in older head and neck cancer patients after radiotherapy. J Clin Oncol 2008;26(31):5119-25.

[8] Dorresteijn LD, Kappelle AC, Boogerd W, et al. Increased risk of ischemic stroke after radiotherapy on the neck in patients younger than 60 years. J Clin Oncol 2002;20(1):282-8.

[9] Rosenthal DI, Fuller CD, Barker JL, et al. Simple carotidsparing intensity-modulated radiotherapy technique and preliminary experience for T1-2 glottic cancer. Int J Radiat Oncol Biol Phys 2010;77(2):455-61.

[10] Janssen S, Glanzmann C, Huber G, et al. Risk-adapted partial larynx and/or carotid artery sparing modulated radiation therapy of glottic cancer. Radiat Oncol 2014;9:136.

[11] Zumsteg ZS, Riaz N, Jaffery S, et al. Carotid sparing intensity-modulated radiation therapy achieves comparable locoregional control to conventional radiotherapy in T1-2N0 laryngeal carcinoma. Oral Oncol 2015;51(7):716-23.

[12] Riegel AC, Antone J, Schwartz DL. Comparative dosimetry of volumetric modulated arc therapy and limited-angle static intensity-modulated radiation therapy for early-stage larynx cancer. Med Dosim 2013;38(1):66-9.

[13] Chakraborty S, Muttath G. Carotid sparing intensitymodulated radiotherapy in early glottic cancers: a case of Maslow's hammer? J Cancer Res Ther 2015;11(2):495-6.

[14] Hong JC, Kruser TJ, Gondi V, et al. Risk of cerebrovascular events in elderly patients after radiation therapy versus surgery for early-stage glottic cancer. Int J Radiat Oncol Biol Phys 2013;87(2):290-6. 